\title{
Honorary Fellows
}

Dr Najeeb Al-Shorbaji

Switzerland

Prof. Ab Bakker

The Netherlands

Dr. Marion J. Ball

USA

Dieter Bergemann

Germany

Lincoln de Assis Moura Jr

Brazil

Prof. Malcolm Forsythe

United Kingdom

Prof. Antoine Geissbuhler

Switzerland

Prof. W. Ed Hammond

USA

Prof. Reinhold Haux

Germany

Prof. Casimir Kulikowski

USA

Dr. Nancy M. Lorenzi

USA
Prof. K.C. Lun

Singapore

Prof. Alexa McCray

USA

Dr. Ramin Moghaddam

Iran

Dr. Peter Murray

United Kingdom

Prof. Otto Rienhoff

Germany

Dr. Jan Roukens

Belgium

Dr. David Shires

USA

Prof. Edward H. Shortliffe

USA

Prof. Hiroshi Takeda

Japan

Prof. Jan H. van Bemmel

The Netherlands

Prof. Valerio Yacubsohn

Argentina

The following IMIA Honorary Fellows have passed away, but we list them in continuing recognition of their contribution to IMIA:

William C. Abbott, United Kingdom

Prof. Branko Cesnik, Australia

Dr. Morris F. Collen, USA

François Grémy, France

Steven A. Huesing, Canada

Dr. Sedick Isaacs, South Africa
Prof. Shigekoto Kaihara, Japan

Prof. Donald A. B. Lindberg, USA

Prof. Hans E. Peterson, Sweden

Mr. Ian H. Symonds, New Zealand

Dr. Jos Willems, Belgium 